\title{
Saturation-Pulse Prepared Heart-Rate Independent Inversion-REcovery (SAPPHIRE) Biventricular T1 Mapping: Inter-Field Strength, Head-To-Head Comparison of Diastolic, Systolic And Dark-Blood Measurements
}

Mashael Alfarih ( $\nabla$ mafarih@iau.edu.sa )

University College London https://orcid.org/0000-0003-3883-314X

João B Augusto

University College London

Kristopher D Knott

University College London

Nasri Fatih

University College London

Praveen Kumar-M

Post Graduate Institute of Medical Education and Research

Redha Boubertakh

Queen Mary University of London Barts and The London School of Medicine and Dentistry

Alun Hughes

University College London

James Moon

University College London

Sebastian Weingärtner

Delft University of Technology: Technische Universiteit Delft

Gabriella Captur

University College London

Research Article

Keywords: T1 mapping, cardiovascular magnetic resonance, SAPPHIRE, MOLLI, dilated cardiomyopathy

Posted Date: August 25th, 2021

DOI: https://doi.org/10.21203/rs.3.rs-834638/v1 
License: (c) (i) This work is licensed under a Creative Commons Attribution 4.0 International License. Read Full License 


\section{Abstract}

Purpose To assess the feasibility of SAPPHIRE $\mathrm{T}_{1}$ mapping in vivo across field strengths and compare results to those obtained by conventional Modified Look-Locker inversion recovery (MOLLI).

Methods 10 healthy volunteers underwent same-day non-contrast cardiovascular magnetic resonance at 1.5 Tesla ( $T$ ) and 3T. Left and right ventricular (LV, RV) $T_{1}$ mapping was performed in the basal, mid and apical short axis using MOLLI and 4-variants of SAPPHIRE: diastolic, systolic, $0^{\text {th }}$ and $2^{\text {nd }}$ order motionsensitized dark blood (DB).

Results LV myocardial $T_{1}$ times differed significantly between MOLLI and each of the SAPPHIRE variants (all $p<0.005)$. LV global myocardial $\mathrm{T}_{1}(1.5 \mathrm{~T}$ then $3 \mathrm{~T}$ results) was significantly longer by diastolic SAPPHIRE $(1283 \pm 11 \mid 1600 \pm 17 \mathrm{~ms})$ than any of the other SAPPHIRE variants: systolic $(1239 \pm 9 \mid 1595 \pm 13 \mathrm{~ms}), 0^{\text {th }}$ order DB $(1241 \pm 10 \mid 1596 \pm 12)$ and $2^{\text {nd }}$ order DB $(1251 \pm 11 \mid 1560 \pm 20 \mathrm{~ms}$, all $p<0.05)$. In the mid septum MOLLI and diastolic SAPPHIRE exhibited significant $T_{1}$ signal contamination (longer $\mathrm{T}_{1}$ ) at the blood-myocardial interface not seen with the other 3 SAPPHIRE variants (all $p<0.025$ ). Additionally, systolic, $0^{\text {th }}$ order and $2^{\text {nd }}$ order DB SAPPHIRE showed narrower dispersion of myocardial $\mathrm{T}_{1}$ times across the mid septum when compared to diastolic SAPPHIRE (interquartile ranges respectively: $25 \mathrm{~ms}, 71 \mathrm{~ms}, 73 \mathrm{~ms}$ vs $143 \mathrm{~ms}$, all $p<0.05)$. RV $\mathrm{T}_{1}$ mapping was achievable using systolic, $0^{\text {th }}$ and $2^{\text {nd }}$ order DB SAPPHIRE but not with MOLLI or diastolic SAPPHIRE. All 4 SAPPHIRE variants showed excellent re-read reproducibility (intraclass correlation coefficients 0.953 to 0.996 ).

Conclusion These preliminary data suggest that systolic and DB SAPPHIRE approaches can reduce myocardial T1 signal contamination by the adjacent bright blood pool at the blood-myocardial interface.

\section{Introduction}

The myocardial longitudinal relaxation time, $T_{1}$ is a sensitive imaging biomarker for heart muscle disease, linked to both functional capacity and mortality [1-5]. Native myocardial $T_{1}$ mapping, that is without the administration of gadolinium-based contrast agents (GBCA), measures this myocardial relaxation time making it a useful imaging biomarker for fibrosis.

The general principle for obtaining $T_{1}$ maps is to acquire multiple images each with a different $T_{1}$ weighting on a longitudinal magnetisation recovery curve. Various techniques have been proposed to quantify $T_{1}$ relaxation (Fig. 1), each with its own advantages and limitations. One of the main issues of commonly-used $\mathrm{T}_{1}$ mapping techniques is the partial volume effect that can artifactually increase the myocardial $\mathrm{T}_{1}$ times of voxels located at the myocardial-blood pool interface due to confounding by the higher native $T_{1}$ signal of the blood pool (consider that typical native myocardial $T_{1}$ is $\sim 1000 \mathrm{~ms}$ while native blood pool $\mathrm{T}_{1}$ is $\sim 1500 \mathrm{~ms}$ at $1.5 \mathrm{Tesla}(\mathrm{T})$ ). This is most apparent in cross sectional imaging and its effects are especially detrimental to the study of thin-walled cardiac structures, notably the right 
ventricular (RV) free wall, atrial walls and the thinned myocardium in dilated cardiomyopathy (DCM) [6, 7]. Partial volume effects are problematic because they reduce the accuracy and reproducibility of $T_{1}$ mapping. To overcome this problem in the right and left ventricles (LV), systolic readouts have been proposed $[8,9]$, as it was hypothesized that the increased myocardial wall thickness during systole would increase the abundance of myocardial voxels that were free from partial volume effects. It follows then that systolic $T_{1}$ mapping would measure lower myocardial $T_{1}$ values at the blood-myocardial interface compared to conventional diastolic $T_{1}$ mapping because of the reduced confounding from the high blood pool signal.

The modified Look-Locker inversion recovery (MOLLI) sequence is still the most commonly used $\mathrm{T}_{1}$ mapping approach in clinical practice due to its widespread availability across vendors and superior precision and reproducibility when compared to most other sequences and prototypes [11]. Being an inversion recovery-based approach, it is vulnerable to the aforementioned partial volume effects, as well as to off-resonance artifacts, and confounding contributions by magnetisation transfer and T2 effects [12]. MOLLI and its derivative, shortened MOLLI (ShMOLLI), have higher precision than the saturation recovery-based $\mathrm{T}_{1}$ mapping technique SAturation-recovery single-SHot Acquisition (SASHA) but are less accurate and prone to underestimate $\mathrm{T}_{1}[13]$.

$A T_{1}$ mapping sequence that combines saturation recovery and inversion recovery approaches, termed SAPPHIRE-SAturation Pulse Prepared Heart-rate independent Inversion REcovery-has recently been shown to have potential advantages in arrhythmia [14]. A further refinement to SAPPHIRE that provides blood suppression resulting in dark-blood (DB) native myocardial $T_{1}$ mapping has shown additional promise at addressing the problem of myocardial signal contamination from the adjacent blood pool [7]. DB SAPPHIRE potentially measures more accurate $T_{1}$ than the conventional diastolic SAPPHIRE because of less contamination from the blood pool signal, but this is at the expense of precision.

Here we undertake a head-to-head comparison of MOLLI versus SAPPHIRE $T_{1}$ mapping across field strengths. We assess the performance of LV and RV SAPPHIRE $\mathrm{T}_{1}$ mapping in vivo considering 4 of its variants: diastolic, systolic, 0th and 2nd order DB SAPPHIRE, and compare results to those obtained by conventional MOLLI across field strengths.

\section{Materials And Methods}

All subjects provided written informed consent. The study received ethical approval from the University College London (UCL) Research Ethics Committee (Project number 6782/001) and it conformed to the principles of the Helsinki Declaration.

\section{Study population and data collection}

In this prospective single-center observational study, 10 healthy volunteers underwent non-contrast CMRI scanning at both field strengths on the same day at the UCL Bloomsbury Center for Clinical Phenotyping 
(London, UK). Volunteers had no prior cardiac history or known cardiac risk factors, were not on cardiovascular medications, had a normal resting electrocardiogram (ECG), and were free of conventional contra-indications for CMRI.

\section{Cardiovascular magnetic resonance}

CMRI studies were performed using two Siemens MR systems (Erlangen, Germany): MAGNETOM AERA 1.5 Tesla (T) (Serial Number 141303) operating VE11C-SP01 and MAGNETOM PRISMA 3T (Serial Number 166032) operating VE11C-SP01, with an 18-channel phased-array chest coil. The scan protocol was identical for the two field strengths and consisted of localizers, transaxial black blood HASTE anatomical stack, breath-held retrospectively ECG-gated balanced steady-state free precession (bSSFP) cines in standard long and short axis views[15], and then breath-held ECG-gated $\mathrm{T}_{1}$ mapping in the basal, mid and apical LV short axis slices (co-registered with the cines) using MOLLI with motion correction (MOCO), bright-blood diastolic SAPPHIRE, bright-blood systolic SAPPHIRE, 0th order DB SAPPHIRE and 2nd order DB SAPPHIRE in random order per scan. To minimize off-resonance artifacts with higher field strengths, the $3 \mathrm{~T}$ protocol additionally included frequency scouts and attention to volumetric shimming.

Typical imaging parameters for the sequences were as follows:

Cine bSSFP 1.5T: Repetition time (TR, time between two consecutive excitations)/ echo time (TE)

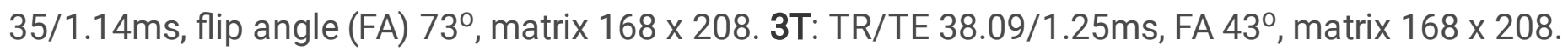

MOLLI 5s(3s)3s 1.5T: TR/TE 291.84/1.22ms, FA 35, matrix 256 x 144, 125 phase-encoding steps, trigger delay (TD) 605ms, slice-thickness 8mm. 3T: TR/TE 283.8/1.16ms, FA 20, matrix 256 x 144, 125 phaseencoding steps, TD 780ms, slice-thickness $8 \mathrm{~mm}$.

Bright-blood diastolic SAPPHIRE 1.5T: TR/TE 830/1.12ms, FA 70, matrix 256 x 192, inversion time (TI) 715ms; images acquired 10; breath-hold duration (@60bpm) 10s; slice-thickness 8mm. 3T: TR/TE 856/1.12ms, FA 68', matrix 256 x 192, TI 740ms; images acquired 10; breath-hold duration (@60bpm) $10 \mathrm{~s}$; slice-thickness $8 \mathrm{~mm}$.

Bright-blood systolic SAPPHIRE 1.5T: TR/TE 830/1.12ms, TD 315ms, FA 70; matrix 256 x 192; images acquired 10; breath-hold duration (@60bpm) 10s; slice-thickness 8mm. 3T: TR/TE 857/1.12ms, TD 392.5ms, FA 68ㅇ, matrix 256 x 192; images acquired 10; breath-hold duration (@60bpm) 10s; slicethickness $8 \mathrm{~mm}$.

$0^{\text {th }}$ order DB SAPPHIRE $1.5 \mathrm{~T}$ : TR/TE $800 / 1.28 \mathrm{~ms}$, TD $677.5 \mathrm{~ms}$, TI $670 \mathrm{~ms}$, FA $70^{\circ}$, matrix $256 \times 192$; images acquired 10; breath-hold duration (@60bpm) 10s; slice-thickness 8mm; MSDE gradient amplitude $20 \mathrm{mT} / \mathrm{m}$ with preparation duration $10 \mathrm{~ms}$. 3T: TR/TE $857 / 1.12 \mathrm{~ms}$, TD $752.5 \mathrm{~ms}$, TI $740 \mathrm{~ms}$, FA $68^{\circ}$, matrix 256 x 192; images acquired 10; breath-hold duration (@60bpm) 10s; slice-thickness 8mm; MSDE gradient amplitude $20 \mathrm{mT} / \mathrm{m}$ with preparation duration $10 \mathrm{~ms}$. 
2nd order DB SAPPHIRE 1.5T: TR/TE 800/1.28ms, TD 677.5ms, TI 670ms, FA 70, matrix 256 x 192; images acquired 10; breath-hold duration (@60bpm) Xs; slice-thickness 8mm; MSDE gradient amplitude $20 \mathrm{mT} / \mathrm{m}$ with preparation duration $10 \mathrm{~ms}$. 3T: TR/TE 857/1.12ms, TD 750ms, TI 740ms, FA 68 ${ }^{\circ}$, matrix 256 x 192; images acquired 10; breath-hold duration (@60bpm) 10s; slice-thickness 8mm; MSDE gradient amplitude $20 \mathrm{mT} / \mathrm{m}$ with preparation duration $10 \mathrm{~ms}$.

\section{Cardiovascular magnetic resonance analysis}

All images were analysed using CVI42 software (Circle Cardiovascular Imaging Inc. v.5.10.1, Calgary, Canada). Measurements were performed by two readers (M.A., J.A.). LV volumes, LV ejection fraction (LVEF) and mass (papillary muscles included in the LV cavity) were determined according to standardized CMRI methods [16] using a semiautomated threshold-based technique and body surface area (BSA) indexation where appropriate. Atrial volumes, LV maximal wall thickness, and mitral and tricuspid annular plane systolic excursions were determined as previously described $[17,18,19]$.

For native $\mathrm{T}_{1}$ measurements in the LV, endo- and epicardial borders were manually drawn per segment according to the 16-segment American Heart Association model, in the 3 short axis slices (10\% offset to avoid the blood-myocardial interface). Segmental $T_{1}$ values were averaged to obtain the mean slice $T_{1}$ and global (whole-heart) $T_{1}$. The global $T_{1}$ was calculated as the mean of basal, mid and apical LV short axis slices.

As demonstrated in Fig. 2 the RV ROI measurement were obtained from the RV inferior wall or free wall in the basal or mid short axis slices if wall thickness $\geq 5 \mathrm{~mm}$.

The transeptal myocardial T1 times for MOLLI and SAPPHIRE variants were calculated using six-points evenly-spaced linear callipers transecting the mid-septal short axis slice on the $T_{1}$ maps of each healthy volunteer (measurements obtained using OsiriX MD).

Intra- (M.A. X 2) and inter-observer (M.A. and J.A.) re-read variability was determined for measurements of average mid slice $T_{1}$, and mid septal region of interest (ROI) $T_{1}$ in 5 randomly chosen CMRI scans ( 2 at $1.5 T$ and 3 at $3 T$ ). Intra-observer variability (M.A. X 2) was performed with one-month temporal interval between repeat analyses.

Native SAPPHIRE $T_{1}$ mapping sequences

\section{A. Diastolic SAPPHIRE}

Conventional diastolic SAPPHIRE (without any attempt to suppress the blood signal, i.e. 'bright blood') consists of a combination of saturation and inversion pulses. A saturation pulse is applied immediately after the $\mathrm{R}$ wave followed by an inversion pulse inserted in the same heartbeat prior to image acquisition (Fig. 3a). The first image acquisition is done without magnetisation preparation. 
Systolic SAPPHIRE also consists of saturation and inversion recovery magnetisation preparation hybrid and 10-ECG triggered readouts but data is acquired during systole. Imaging in systole is challenging due to the short time window available between $R$ waves resulting in a weak saturation recovery $T_{1}$ mapping signal. As a fix, saturation is performed in the preceding heart-beat directly following the imaging pulses played in the previous heart-beat. Similar to diastolic SAPPHIRE, the first image is acquired without magnetisation preparation. The remaining images are obtained with an extra inversion pulse with variable delay following the $\mathrm{R}$ wave (Fig. $3 b$ ).

\section{Oth and 2nd Order dark blood diastolic SAPPHIRE}

Dark blood $\mathrm{T}_{1}$ mapping is achieved using a modified SAPPHIRE technique [10]. For blood suppression, motion sensitized driven equilibrium (MSDE) preparation is inserted before the bSSFP imaging readout (Fig. 4a). The MSDE preparation consists of a $90^{\circ}$ excitation tip-down pulse, a one or more $180^{\circ}$ refocusing pulse, and a $-90^{\circ}$ flip-back pulse to encode the spin dephasing in the longitudinal magnetisation (Fig. 4a). Strong motion sensitising gradients are also sandwiched in between the radiofrequency pulses to induce dephasing. Two kinds of motion sensitising gradients are employed, with nulling the gradient moment up to the 0th and 2nd order respectively. To achieve 0th order gradient nulling, identical trapezoidial gradients are played before and after the refocusing pulse.

Additionally, in this study advanced motion sensitising gradients were introduced. As illustrated in Fig. 4b, reverse bipolar gradient blips are inserted to achieve 0th and 2nd moment nulling.

\section{Data analysis and statistics}

Statistical analysis was performed in R programming language (version 3.6.0, The R Foundation for Statistical Computing) and SPSS statistical software (version 26.0, IBM Corp., Armonk, NY, USA). Descriptive data are expressed as mean \pm standard deviation except where otherwise stated. The distribution of data was evaluated by histograms and Shapiro-Wilk test. Parametric and nonparametric continuous variables pertaining to participants were compared using student $t$-test or Mann-Whitney $\mathrm{U}$ test as appropriate. Categorical variables were compared by $\chi^{2}$ or Fisher's exact tests.

Linear mixed effect models were used to compare T1 times across SAPPHIRE techniques (fixed effects: e.g. systolic vs. diastolic, 1.5T vs. 3T, slice level) accounting for repeatedness (participant number). In addition, paired-samples $t$-test (parametric) or related-samples Wilcoxon signed rank test (nonparametric) were used for pairwise comparisons between MOLLI and SAPPHIRE, and within SAPPHIRE, across field strengths using Bonferroni correction.

Differences in transeptal $T_{1}$ mapping profiles between sequences were assessed using two samples Anderson-Darling test as it gives more weight to the tails of the distribution (that is close to the bloodmyocardial interface which was of particular interest to us). Two-sided $p$-values $<0.05$ were considered significant. 
Intra- and inter-observer variability (absolute agreement) of T1 mapping values was assessed using twoway random, single measures intraclass correlation coefficient (ICC).

\section{Results}

\section{Study population characteristics}

Demographic and CMRI characteristics of the 10 healthy volunteers ( 8 females, age $36 \pm 10$ years) are presented in Table 1.

\section{Feasibility in vivo}

SAPPHIRE $T_{1}$ mapping using all 4 variants was completed successfully in all subjects (Tables $2 \& 3$ ). ROI placement for RV T1 mapping was not feasible using MOLLI or diastolic SAPPHIRE because of insufficient wall thickness, but it was possible using systolic, 0th order and 2nd order DB SAPPHIRE and results are reported in Table 3 .

\section{Inter-field strength differences}

Healthy volunteer native myocardial $\mathrm{T}_{1}$ values obtained by MOLLI and the 4 SAPPHIRE variants across field strengths are reported in Table 2 and global LV $T_{1}$ times provided in Fig. 5. As expected $T_{1}$ values within sequences were consistently higher at $3 \mathrm{~T}$ than at $1.5 \mathrm{~T}$ (all $p<0.005$, Fig. 6).

\section{Differences between MOLLI and SAPPHIRE variants}

Pairwise comparisons showed that at both field strengths MOLLI measured significantly lower LV global myocardial $\mathrm{T}_{1}$ times than any of the other 4 SAPPHIRE variants (all $p<0.005$, Online Resource 1, Table 1). These differences persisted after removing the apical slice data (Online Resource 1, Table 2, $p<0.005$ ).

\section{Differences between SAPPHIRE variants}

Using linear mixed models to adjust for field strength, phase, slice location and subject, myocardial $T_{1}$ times by diastolic SAPPHIRE were significantly longer than with systolic, 0th order DB and 2nd order DB SAPPHIRE at 1.5T and significantly longer than with systolic and 2nd order DB SAPPHIRE at 3T (all $p<$ 0.05 , Fig. 7a). However, after excluding the apical slice the myocardial $\mathrm{T}_{1}$ times by diastolic SAPPHIRE were significantly longer than with systolic, 0th order DB and 2nd order DB SAPPHIRE at both field strengths (all $p<0.05$, Fig. $7 b$ ).

At 1.5T pairwise comparisons for $L V$ global $T_{1}$ showed that diastolic SAPPHIRE measured significantly longer $\mathrm{T}_{1}$ times than the 0th order DB SAPPHIRE ( $p=0.014$, Online Resource 1,Table 1$)$ but not after excluding the apical slice data (Online Resource 1, Table 2). Pairwise comparisons of RV $\mathrm{T}_{1}$ times showed no differences between DB SAPPHIRE variants (all $p>0.005$, Online Resource 1, Table 3). At 3T 
systolic SAPPHIRE measured significantly longer $\mathrm{RV} \mathrm{T}_{1}$ times than the 0th order and 2 nd order SAPPHIRE ( $p=0.028$ and 0.001 , respectively) but no such differences were observed at $1.5 \mathrm{~T}$ (all $p>0.05$ )

\section{Transmural $T_{1}$ mapping profiles of MOLLI and SAPPHIRE variants}

Transmural $\mathrm{T}_{1}$ times (Fig. $8 b$ ) in the mid septum immediately adjacent to the LV and RV blood pools were longer by MOLLI and diastolic SAPPHIRE when compared to systolic, 0th order DB and 2nd order DB SAPPHIRE. Contamination from the high $T_{1}$ of the blood pool appeared as an upsloping $T_{1}$ profile near the edges of the septal profile for both MOLLI and diastolic SAPPHIRE sequences, but not for the other SAPPHIRE variants. Indeed, the profile distributions of transmural myocardial $\mathrm{T}_{1}$ times by the AndersonDarling test differed significantly between MOLLI and all 4 SAPPHIRE variants (diastolic $p=0.0003$; systolic $p=0.0003$; 0th order DB $p=0.024 ; 2$ nd order DB $p=0.025$ ); between diastolic SAPPHIRE and both DB variants (0th order DB $p=0.0003 ; 2$ nd order DB $p=0.001$ ); and between systolic SAPPHIRE and both DB variants (0th order DB $p=0.0003$; 2nd order DB $p=0.0005$ ), Furthermore, the dispersion of transmural $\mathrm{T}_{1}$ times across the mid septum for systolic $(1233.3 \pm 21.6 \mathrm{~ms}$; interquartile range [IQR] $=25.3 \mathrm{~ms})$ and $\mathrm{DB}$ SAPPHIRE variants (0th order: $1205.8 \pm 46.1 \mathrm{~ms}$; IQR $=71.0 \mathrm{~ms} \mid 2$ nd order: $1177.5 \pm 51.9 \mathrm{~ms}$; IQR $=72.9 \mathrm{~ms}$ ) was narrower when compared to diastolic SAPPHIRE $(1263.3 \pm 85.6 \mathrm{~ms}$; IQR $=142.8 \mathrm{~ms})$, and the dispersion for systolic SAPPHIRE narrower than of MOLLI ( $1054 \pm 42.2 \mathrm{~ms}$; IQR $=56.4 \mathrm{~ms})$.

Re-read variability of $T_{1}$ mapping measurements

Intra- and interobserver variability of myocardial $\mathrm{T}_{1}$ reads was excellent across all sequences tested with ICCs ranging from 0.953-0.996 (Table 4).

\section{Discussion}

This study sought to compare native myocardial $\mathrm{T}_{1}$ mapping values of both ventricles by MOLLI against those obtained by diastolic, systolic and dark blood SAPPHIRE variants, across field strengths. As expected MOLLI measured longer myocardial $\mathrm{T}_{1}$ times than the conventional diastolic SAPPHIRE sequence [13]. We found that native $T_{1}$ was significantly shorter by the systolic, 0 th order $D B$ and $2 n d$ order DB SAPPHIRE variants compared to diastolic SAPPHIRE across field strengths. We go on to show that systolic and DB SAPPHIRE variants reduce the dispersion of trans-myocardial $T_{1}$ times across the septum and abolish the artefactual $\mathrm{T}_{1}$ lengthening in voxels located at the blood-myocardial/epicardial boundaries that remains a visible problem for both MOLLI and diastolic SAPPHIRE. Combined, these data suggest that systolic and DB SAPPHIRE approaches may help counteract the problem of partial volume effects. According to these data, the 0th and 2nd order DB SAPPHIRE sequences appear to be equivalent at preventing myocardial $T_{1}$ signal contamination by the adjacent blood pool. Systolic SAPPHIRE was the sequence that produced the narrowest dispersion of myocardial $\mathrm{T}_{1}$ times across the mid septum (even narrower than MOLLI). 
Previous work at $3 T$ has suggested that native myocardial $T_{1}$ in health lengthens progressively from base to apex $[20,21]$ and we observed a similar trend by MOLLI at $3 \mathrm{~T}$ but not at $1.5 \mathrm{~T}$, and not with the majority of SAPPHIRE variants used in this study. The most likely explanation for the lengthening $T_{1}$ from base to apex is that partial volume effects are more prevalent in the thinner more apical segments, and exacerbated by the natural curvature of the apical cap.

Results from linear mixed model analysis showing that native myocardial $T_{1}$ by diastolic SAPPHIRE was significantly longer than by systolic SAPPHIRE at both field strengths, are in agreement with previous work $[8,12,22-23]$. It has been postulated that in systolic $T_{1}$ mapping these differences were due to reduced partial volume effects thanks to the increased LV wall thickness. The fact that 0th order and 2nd order DB SAPPHIRE $T_{1}$ times were significantly shorter than diastolic SAPPHIRE suggests that blood pool suppression is another potential solution for the problem of partial volume effects.

We observed that the RV free wall was visible and indeed analysable on the short axis cine slices by systolic and DB SAPPHIRE approaches but hardly visible at all by conventional bright blood SAPPHIRE or MOLLI. We found that $R V T_{1}$ was significantly longer than $L V T_{1}$ which might be due to residual partial voluming and/or the naturally higher collagen content of the RV [24].

The published literature provides conflicting data on the relationship between $R V$ and $L V T_{1}$, with groups reporting higher $\mathrm{RV} \mathrm{T}_{1}$ [25-27], lower $\mathrm{RV} \mathrm{T}_{1}$ [28] or equivalent $\mathrm{RV} / \mathrm{LV} \mathrm{T}_{1}$ reads [29] in various cohorts, not all of which were healthy controls as in our case. Although we took care to draw precise ROIs in the RV myocardium, partial voluming and contamination of $R V T_{1}$ times from the inadvertent inclusion of voxels contaminated by blood pool or epicardial fat signals are plausible pitfalls that could have artefactually lengthened our native RV myocardial $\mathrm{T}_{1}$ times.

Overall, the evidence presented in this study suggests that systolic and DB approaches are less prone to partial volume effects than MOLLI and diastolic SAPPHIRE. The transeptal myocardial $T_{1}$ times immediately adjacent to LV/RV blood pools recorded for 0th and 2nd order DB SAPPHIRE were significantly lower than those recorded for systolic SAPPHIRE (Fig. 8b) suggesting that blood pool nulling achieved by the $\mathrm{DB} \mathrm{T}_{1}$ mapping approaches provides some additional benefit thanks to the reduced sensitivity to partial volume effects. Future work should explore the potential clinical utility of DB SAPPHIRE $T_{1}$ mapping in the thin-walled hearts of patients with dilated or arrhythmogenic right ventricular cardiomyopathy.

We report excellent intra- and inter-observer re-read reproducibility for all the methods studied with all ICCs (>0.90) being well in line with previous reports $[7,20,22,23,30-33]$.

In this study we also demonstrate the use of higher order gradient moment nulling for dark blood $\mathrm{T}_{1}$ mapping. In previous literature 0th order nulling was found to be susceptible to residual myocardial motion in some cases, necessitating fine tuning of the motion sensitising gradient moment. As the residual cardiac motion is less turbulent compared to blood flow, stronger motion sensitizing gradients 
may be applied when nulling the gradient moment up to the 2nd order. Even though quantification results were comparable between 0th and 2nd order DB SAPPHIRE in our experiments, 2nd order nulling may have the advantage of increased ease of use for clinical translation. Robustness of higher order gradient nulling for $\mathrm{DB} \mathrm{T}_{1}$ mapping, thus, warrants further investigation in a patient cohort.

\section{Limitations}

Study limitations include the small healthy volunteer cohort size and single-center, single-vendor study design. Comparison to other $\mathrm{T}_{1}$ mapping sequences (such as ShMOLLI and SAturation recovery singleSHot Acquisition [SASHA]) was not undertaken and neither was post-GBCA $\mathrm{T}_{1}$ mapping. Future work will need to examine the clinical utility of these SAPPHIRE sequences in patients with arrhythmia, dilated cardiomyopathy and for the study of other thin-walled cardiac structures such as the atria. RV $T_{1}$ analysis was not possible on MOLLI or diastolic SAPPHIRE due to the RV thickness limitation. MOCO was used for MOLLI sequences but not for the SAPPHIRE $T_{1}$ mapping sequences. Future work should seek to assess the impact of heart rate variability on the spin dynamic in the systolic SAPPHIRE sequence.

In conclusion these preliminary healthy volunteer data suggest that systolic and dark blood approaches can reduce myocardial $\mathrm{T}_{1}$ signal contamination by the adjacent bright blood pool at the blood-myocardial interface-a problem which still hampers other widely-used conventional $\mathrm{T}_{1}$ mapping approaches in clinical practice.

\section{Declarations}

\section{Funding}

This study was funded by the 2017 Society of Cardiovascular Magnetic Resonance Seed Grant Award to G.C. M.A. is supported by Saudi Arabian Cultural Bureau in London. G.C. is supported by the British Medical Association Josephine Lansdell research grant, by the British Heart Foundation (MyoFit46 Special Programme Grant SP/20/2/34841), the National Institute for Health Research Rare Diseases Translational Research Collaboration (NIHR RD-TRC) and by the NIHR UCL Hospitals Biomedical Research Center. J.C.M. is directly and indirectly supported by the UCL Hospitals NIHR BRC and Biomedical Research Unit at Barts Hospital respectively. S.W. acknowledges grant support by the 4TU Federation, NWO Startup and ZonMW OffRoad.

\section{Competing interests}

The authors declare that they have no competing interests

\section{Availability of data and materials}

The datasets used and/or analysed during the current study are available from the corresponding author on reasonable request. 


\section{Code availability}

Not applicable

\section{Authors' contributions}

M.A. analysed the data, performed the statistical analysis and wrote the manuscript. J.B.A., K.D.K. and G.C. collected the data. J.B.A., K.D.K., N.F., P.K.M. and S.W. contributed to data analysis and review of the manuscript. R.B., A.D.H. and J.C.M. provided expert review of the manuscript. G.C., S.W. and J.C.M. conceived of the project.

\section{Ethics approval and consent to participate}

The study received ethical approval from the University College London (UCL) Research Ethics Committee.

\section{Consent for publication}

All subjects provided written informed consent

\section{Acknowledgements}

Not applicable

\section{References}

[1] Dweck MR, Joshi S, Murigu T, et al. Midwall fibrosis is an independent predictor of mortality in patients with aortic stenosis. J Am Coll Cardiol 2011;58:1271-1279.

[2] Gulati A, Jabbour A, Ismail TF, et al. Association of fibrosis with mortality and sudden cardiac death in patients with nonischemic dilated cardiomyopathy. JAMA 2013;309:896-908.

[3] Wong TC, Piehler K, Meier CG, et al. Association between extracellular matrix expansion quantified by cardiovascular magnetic resonance and short-term mortality. Circulation 2012;126:1206-1216.

[4] Wong TC, Piehler KM, Kang IA, et al. Myocardial extracellular volume fraction quantified by cardiovascular magnetic resonance is increased in diabetes and associated with mortality and incident heart failure admission. Eur Heart J 2014;35:657-664.

[5] Vassiliou VS, Perperoglou A, Raphael CE, et al. Midwall fibrosis and 5-year outcome in patients with moderate and severe aortic stenosis. J Am Coll Cardiol 2017;69:1755-1756.

[6] Kellman P, Hansen MS. T1-mapping in the heart: accuracy and precision. J Cardiovasc Magn Reson 2014;16:2-12. 
[7] Weingärtner S, Meßner NM, Zöllner FG, Akçakaya M, Schad LR. Black-blood native T1mapping: Blood signal suppression for reduced partial voluming in the myocardium. Magn Reson Med 2017;78:484-493.

[8] Ferreira VM, Wijesurendra RS, Liu A, Greiser A, Casadei B, Robson MD, Neubauer S, Piechnik SK. Systolic ShMOLLI myocardial T1-mapping for improved robustness to partial-volume effects and applications in tachyarrhythmias. J Cardiovasc Magn Reson 2015;17:77-85.

[9] Meßner NM, Budjan J, Loßnitzer D, Papavassiliu T, Schad LR, Weingärtner S, Zöllner FG. Saturationrecovery myocardial t1-mapping during systole: accurate and robust quantifcation in the presence of arrhythmia. Sci Rep 2018;8:5251-5260.

[10] Weingärtner S, Akçakaya M, Basha T, et al. Combined saturation/inversion recovery sequences for improved evaluation of scar and diffuse fibrosis in patients with arrhythmia or heart rate variability. Magn Reson Med 2014;71:1024-1034.

[11] Captur G, Bhandari A, Brühl R, et al. T1 mapping performance and measurement repeatability: results from the multi-national T1 mapping standardization phantom program (T1MES). J Cardiovasc Magn Reson 2020;22:31-41.

[12] Zhao L, Li S, Ma X, Greiser A, et al. Systolic MOLLI T1 mapping with heart-rate-dependent pulse sequence sampling scheme is feasible in patients with atrial fibrillation. $\mathrm{J}$ Cardiovasc Magn Reson 2016;18:13-23.

[13] Roujol S, Weingärtner S, Foppa M, et al. Accuracy, precision, and reproducibility of four T1 mapping sequences: a head-to-head comparison of MOLLI, ShMOLLI, SASHA, and SAPPHIRE. Radiology 2014;272:683-689.

[14] Wang Y, Vidan E, Bergman GW. Cardiac motion of coronary arteries: variability in the rest period and implications for coronary MR angiography. Radiology 1999;213:751-758.

[15] Kramer CM, Barkhausen J, Bucciarelli-Ducci C, Flamm SD, Kim RJ, Nagel E. Standardised cardiovascular magnetic resonance (CMR) protocols 2013 update. J Cardiovasc Magn Reson 2013;15:91-110.

[16] Alfakih K, Plein S, Thiele H, Jones T, Ridgway JP, Sivananthan MU. Normal human left and right ventricular dimensions for MRI as assessed by turbo gradient echo and steady-state free precession imaging sequences. J Magn Reson Imaging 2003;17:323-329.

[17] Doesch C, Lossnitzer D, Rudic B, et al. Right ventricular and right atrial involvement can predict atrial fibrillation in patients with hypertrophic cardiomyopathy? Int J Med Sci 2016;13:1-7.

[18] Zareian M, Ciuffo L, Habibi M, et al. Left atrial structure and functional quantitation using cardiovascular magnetic resonance and multimodality tissue tracking: validation and reproducibility assessment. J Cardiovasc Magn Reson 2015;17:52-56. 
[19] Captur G, Lopes LR, Patel V, et al. Abnormal cardiac formation in hypertrophic cardiomyopathy: fractal analysis of trabeculae and preclinical gene expression. Circ Cardiovasc Genet 2014;7:241-248.

[20] von Knobelsdorff-Brenkenhoff F, Prothmann M, Dieringer MA, et al. Myocardial T1 and T2 mapping at 3T: reference values, influencing factors and implications. J Cardiovasc Magn Reson 2013;15:53-60.

[21] Dong $Y$, Yang D, Han $Y$, et al. Age and gender impact the measurement of myocardial interstitial fibrosis in a healthy adult chinese population: a cardiac magnetic resonance study. Front Physiol 2018;9:140-150.

[22] Kawel N, Nacif M, Zavodni A, Jones J, Liu S, Sibley CT, Bluemke DA. T1 mapping of the myocardium: intra-individual assessment of the effect of field strength, cardiac cycle and variation by myocardial region. J Cardiovasc Magn Reson 2012;14:27-35.

[23] Reiter U, Reiter G, Dorr K, Greiser A, Maderthaner R, Fuchsjäger M. Normal diastolic and systolic myocardial T1 values at 1.5-T MR imaging: correlations and blood normalization. Radiology 2014;271:365-372.

[24] Oken DE, Boucek RJ. Quantitation of collagen in human myocardium. Circ Res 1957;5:357-361.

[25] Kawel-Boehm N, Dellas Buser T, Greiser A, Bieri O, Bremerich J, Santini F. In-vivo assessment of normal T1 values of the right-ventricular myocardium by cardiac MRI. Int J Cardiovasc Imaging 2014;30:323-328.

[26] Secchi F, Alì M, Monti CB, Greiser A, Pluchinotta FR, Carminati M, Sardanelli F. Right and left ventricle native T1 mapping in systolic phase in patients with congenital heart disease. Acta Radiol 2021;62:334340 .

[27] Karur GR, Robison S, Iwanochko RM, et al. Use of myocardial t1 mapping at $3.0 \mathrm{t}$ to differentiate anderson-fabry disease from hypertrophic cardiomyopathy. Radiology 2018;288:398-406.

[28] Wang J, Zhao H, Wang Y, Herrmann HC, Witschey WRT, Han Y. Native T1 and T2 mapping by cardiovascular magnetic resonance imaging in pressure overloaded left and right heart diseases. $J$ Thoracic Dis 2018;10:2968-2975.

[29] Mehta BB, Auger DA, Gonzalez JA, et al. Detection of elevated right ventricular extracellular volume in pulmonary hypertension using Accelerated and Navigator-Gated Look-Locker Imaging for Cardiac T1 Estimation (ANGIE) cardiovascular magnetic resonance. J Cardiovasc Mag Res 2015;17:110-120.

[30] Chin CW, Semple S, Malley T, et al. Optimization and comparison of myocardial T1 techniques at 3T in patients with aortic stenosis. Eur Heart J Cardiovasc Imaging 2014;15:556-565.

[31] Puntmann VO, Voigt T, Chen Z, et al. Native T1 mapping in differentiation of normal myocardium from diffuse disease in hypertrophic and dilated cardiomyopathy. JACC Cardiovasc Imaging 2013;6:475- 
84.

[32] Vassiliou V, Heng EL, Sharma P, et al. Reproducibility of T1 mapping 11-heart beat MOLLI Sequence. J Cardiovasc Magn Reson 2015;17:W26.

[33] Weingärtner S, Meßner NM, Budjan J, et al. Myocardial T1-mapping at 3T using saturation-recovery: reference values, precision and comparison with MOLLI. J Cardiovasc Magn Reson 2017;18:84-90.

\section{Tables}


Table 1

Demographic and CMRI characteristics of the healthy volunteers.

\section{Healthy Volunteers $(n=10)$}

Demographics

Age, years

$36 \pm 10$

Female

$8(80 \%)$

Height, $\mathrm{cm}$

$168.4 \pm 8.7$

Weight, kg

$71.6 \pm 13.3$

BSA, $m^{2}$

$1.8 \pm 0.2$

CMRI parameters

$\mathrm{LAVi}, \mathrm{mL} / \mathrm{m}^{2}$

$11.0 \pm 2.1$

$\mathrm{RAVi}, \mathrm{mL} / \mathrm{m}^{2}$

$11.3 \pm 1.3$

LVEDVi, $\mathrm{mL} / \mathrm{m}^{2}$

$74.6 \pm 0.2$

LVESVi, $\mathrm{mL} / \mathrm{m}^{2}$

$27.6 \pm 0.2$

LVEF, \%

$63 \pm 5.1$

MAPSE, $\mathrm{mm}$

$15.3 \pm 2.7$

LV MWT, mm

$7.8 \pm 1.0$

LVMi, $\mathrm{g} / \mathrm{m}^{2}$

$48.3 \pm 12.5$

RVEDVi, $\mathrm{mL} / \mathrm{m}^{2}$

$75.1 \pm 9.0$

RVESVi, $\mathrm{mL} / \mathrm{m}^{2}$

$38.6 \pm 9.9$

RVEF, \%

$49 \pm 10.2$

RVMi, $\mathrm{g} / \mathrm{m}^{2}$

TAPSE, $\mathrm{mm}$

Data reported as mean \pm 1 SD or count (\%) as appropriate.

BSA, body surface area; CMRI, cardiovascular magnetic resonance imaging; LAVi, left atrial volume; LVEDVi, left ventricular end-diastolic volume indexed to body surface area; LVEF, left ventricular ejection fraction; LVESVi, left ventricular end-systolic volume indexed to body surface area; LVMi, left ventricular mass; MAPSE, mitral annular plane systolic excursion; MWT, maximum wall thickness; RAVi, right atrial volume; RVEDVi, right ventricular end-diastolic volume indexed to body surface area; RVEF, right ventricular ejection fraction; RVESVi, right ventricular end-systolic volume indexed to body surface area; SD, standard deviation; TAPSE, tricuspid annular plane systolic excursion. 
Table 2

Summary of $L V T_{1}$ mapping data by MOLLI and the 4 SAPPHIRE variants across field strengths.

\begin{tabular}{|c|c|c|c|c|c|c|c|}
\hline Sequence & $\begin{array}{l}\text { Field } \\
\text { Strength }\end{array}$ & $\begin{array}{l}\text { Basal } \\
\text { SAX }\end{array}$ & $\begin{array}{l}\text { Mid } \\
\text { SAX }\end{array}$ & $\begin{array}{l}\text { Apical } \\
\text { SAX }\end{array}$ & $\begin{array}{l}\text { Mid } \\
\text { Septal } \\
\text { ROI }\end{array}$ & $\begin{array}{l}\text { Global } \\
T_{1}\end{array}$ & $\begin{array}{l}\text { Non-apical } \\
\text { Global } T_{1}\end{array}$ \\
\hline \multirow[t]{2}{*}{ MOLLI } & $1.5 \mathrm{~T}$ & $\begin{array}{l}1032.1 \\
\pm 22.9\end{array}$ & $\begin{array}{l}1028.8 \\
\pm 25.8\end{array}$ & $\begin{array}{l}1029.6 \\
\pm 20.9\end{array}$ & $\begin{array}{l}1037.3 \pm \\
27.7\end{array}$ & $\begin{array}{l}1034.1 \\
\pm 6.7\end{array}$ & $1031.3 \pm 2.3$ \\
\hline & $3 T$ & $\begin{array}{l}1297.2 \\
\pm 26.7\end{array}$ & $\begin{array}{l}1296.7 \\
\pm 25.5\end{array}$ & $\begin{array}{l}1310.7 \\
\pm 31.9\end{array}$ & $\begin{array}{l}1309.9 \pm \\
36.4\end{array}$ & $\begin{array}{l}1298.2 \\
\pm 8.8\end{array}$ & $1295.4 \pm 2.5$ \\
\hline \multirow[t]{2}{*}{$\begin{array}{l}\text { Diastolic } \\
\text { SAPPHIRE }\end{array}$} & $1.5 \mathrm{~T}$ & $\begin{array}{l}1261.4 \\
\pm 45.2\end{array}$ & $\begin{array}{l}1266.7 \\
\pm 33.3\end{array}$ & $\begin{array}{l}1341.9 \\
\pm 59.9\end{array}$ & $\begin{array}{l}1249.6 \pm \\
79.4\end{array}$ & $\begin{array}{l}1283.6 \\
\pm 10.5\end{array}$ & $1264.1 \pm 3.1$ \\
\hline & $3 T$ & $\begin{array}{l}1636.5 \\
\pm 83\end{array}$ & $\begin{array}{l}1596.2 \\
\pm 48.4\end{array}$ & $\begin{array}{l}1593.3 \\
\pm 70.1\end{array}$ & $\begin{array}{l}1602.5 \pm \\
52.7\end{array}$ & $\begin{array}{l}1600.7 \\
\pm 17.3\end{array}$ & $1612.2 \pm 5.7$ \\
\hline \multirow[t]{2}{*}{$\begin{array}{l}\text { Systolic } \\
\text { SAPPHIRE }\end{array}$} & $1.5 \mathrm{~T}$ & $\begin{array}{l}1285.9 \\
\pm 51.1\end{array}$ & $\begin{array}{l}1225.4 \\
\pm 21\end{array}$ & $\begin{array}{l}1238.9 \\
\pm 18\end{array}$ & $\begin{array}{l}1224.7 \pm \\
41.8\end{array}$ & $\begin{array}{l}1238.6 \\
\pm 8.9\end{array}$ & $1252.7 \pm 4.9$ \\
\hline & $3 T$ & $\begin{array}{l}1580.1 \\
\pm 40.5\end{array}$ & $\begin{array}{l}1586.3 \\
\pm 44.2\end{array}$ & $\begin{array}{l}1596.3 \\
\pm 43.4\end{array}$ & $\begin{array}{l}1584.7 \pm \\
47.6\end{array}$ & $\begin{array}{l}1595.7 \\
\pm 12.9\end{array}$ & $1590.6 \pm 4.2$ \\
\hline \multirow[t]{2}{*}{$\begin{array}{l}\text { DB 0th order } \\
\text { SAPPHIRE }\end{array}$} & $1.5 \mathrm{~T}$ & $\begin{array}{l}1228.5 \\
\pm 57\end{array}$ & $\begin{array}{l}1247.8 \\
\pm 29.7\end{array}$ & $\begin{array}{l}1245.9 \\
\pm 54.7\end{array}$ & $\begin{array}{l}1227.8 \pm \\
78.2\end{array}$ & $\begin{array}{l}1240.9 \\
\pm 9.7\end{array}$ & $1242.4 \pm 2.9$ \\
\hline & $3 T$ & $\begin{array}{l}1570.9 \\
\pm 68.6\end{array}$ & $\begin{array}{l}1595.2 \\
\pm 45.5\end{array}$ & $\begin{array}{l}1607.5 \\
\pm 42.3\end{array}$ & $\begin{array}{l}1596.7 \pm \\
73.9\end{array}$ & $\begin{array}{l}1596.4 \\
\pm 11.9\end{array}$ & $1583.7 \pm 4.7$ \\
\hline \multirow[t]{2}{*}{$\begin{array}{l}\text { DB 2nd order } \\
\text { SAPPHIRE }\end{array}$} & $1.5 \mathrm{~T}$ & $\begin{array}{l}1234.6 \\
\pm 46.1\end{array}$ & $\begin{array}{l}1225.3 \\
\pm 61\end{array}$ & $\begin{array}{l}1297.7 \\
\pm 50.6\end{array}$ & $\begin{array}{l}1220.1 \pm \\
110.6\end{array}$ & $\begin{array}{l}1251.2 \\
\pm 10.9\end{array}$ & $1237.2 \pm 4.6$ \\
\hline & $3 T$ & $\begin{array}{l}1549.6 \\
\pm 64.9\end{array}$ & $\begin{array}{l}1554.6 \\
\pm 63.9\end{array}$ & $\begin{array}{l}1569.1 \\
\pm 31.3\end{array}$ & $\begin{array}{l}1503.2 \pm \\
132.3\end{array}$ & $\begin{array}{l}1560.6 \\
\pm 20.2\end{array}$ & $1560.5 \pm 4.1$ \\
\hline \multicolumn{8}{|c|}{$\begin{array}{l}\text { Values reported are mean } \pm 1 \text { SD. } \\
\text { DB, Dark blood; MOLLI, Modified Look-Locker inversion recovery; ROI, region of interest; SAPPHIRE, } \\
\text { SAturation Pulse Prepared Heart-rate independent Inversion REcovery; SAX, short axis; T, Tesla. Other } \\
\text { abbreviations as in Table } 1 .\end{array}$} \\
\hline
\end{tabular}


Table 3

Summary of RV $T_{1}$ mapping values by MOLLI and the 4 SAPPHIRE variants across field strengths

\begin{tabular}{|c|c|c|c|c|c|}
\hline Sequence & $\begin{array}{l}\text { Field } \\
\text { Strength }\end{array}$ & $\begin{array}{l}\text { Basal SAX (inferior or } \\
\text { free wall) }\end{array}$ & $\begin{array}{l}\text { Mid SAX } \\
\text { (inferior } \\
\text { wall) }\end{array}$ & $\begin{array}{l}\text { Mid SAX } \\
\text { (free wall) }\end{array}$ & $\begin{array}{l}\text { Global T1 } \\
\text { ROI }\end{array}$ \\
\hline \multirow[t]{2}{*}{ MOLLI* } & $1.5 \mathrm{~T}$ & NA & NA & NA & NA \\
\hline & $3 \mathrm{~T}$ & NA & NA & NA & NA \\
\hline \multirow{2}{*}{$\begin{array}{l}\text { Diastolic } \\
\text { SAPPHIRE* }\end{array}$} & $1.5 \mathrm{~T}$ & NA & NA & NA & NA \\
\hline & $3 T$ & NA & NA & NA & NA \\
\hline \multirow[t]{2}{*}{$\begin{array}{l}\text { Systolic } \\
\text { SAPPHIRE }\end{array}$} & $1.5 \mathrm{~T}$ & $1530.9 \pm 93.9$ & $\begin{array}{l}1479.3 \pm \\
97.9\end{array}$ & $\begin{array}{l}1421.5 \pm \\
57.5\end{array}$ & $\begin{array}{l}1477.2 \pm \\
83.1\end{array}$ \\
\hline & $3 T$ & $1766.2 \pm 14.0$ & $\begin{array}{l}1757.3 \pm \\
51.4\end{array}$ & $\begin{array}{l}1769.0 \pm \\
31.2\end{array}$ & $\begin{array}{l}1764.2 \pm \\
32.2\end{array}$ \\
\hline \multirow[t]{2}{*}{$\begin{array}{l}\text { DB 0th order } \\
\text { SAPPHIRE }\end{array}$} & $1.5 \mathrm{~T}$ & $1439.6 \pm 67.3$ & $\begin{array}{l}1453.0 \pm \\
122.7\end{array}$ & $\begin{array}{l}1452.6 \pm \\
104.4\end{array}$ & $\begin{array}{l}1448.4 \pm \\
98.1\end{array}$ \\
\hline & $3 \mathrm{~T}$ & $1723.8 \pm 37.1$ & $\begin{array}{l}1680.5 \pm \\
43.0\end{array}$ & $\begin{array}{l}1688.8 \pm \\
49.0\end{array}$ & $\begin{array}{l}1697.7 \pm \\
43.0\end{array}$ \\
\hline \multirow[t]{2}{*}{$\begin{array}{l}\text { DB 2nd order } \\
\text { SAPPHIRE }\end{array}$} & $1.5 \mathrm{~T}$ & $1523.9 \pm 44.3$ & $\begin{array}{l}1416.9 \pm \\
81.5\end{array}$ & $\begin{array}{l}1452.4 \pm \\
54.0\end{array}$ & $\begin{array}{l}1464.4 \pm \\
60.0\end{array}$ \\
\hline & $3 T$ & $1684.0 \pm 101.3$ & $\begin{array}{l}1666.8 \pm \\
63.0\end{array}$ & $\begin{array}{l}1657.9 \pm \\
77.5\end{array}$ & $\begin{array}{l}1669.5 \pm \\
80.6\end{array}$ \\
\hline \multicolumn{6}{|c|}{$\begin{array}{l}\text { Values reported are mean } \pm 1 \text { SD. } \\
\text { * RV measurements were obtained only when the RV wall thickness } \geq 5 \mathrm{~mm} \text { and this was not } \\
\text { achievable with MOLLI or diastolic SAPPHIRE. } \\
\text { DB, Dark blood; MOLLI, Modified Look-Locker inversion recovery; ROI, region of interest; RV, right } \\
\text { ventricle; SAPPHIRE, SAturation Pulse Prepared Heart-rate independent Inversion REcovery; SAX, short } \\
\text { axis; T, Tesla. Other abbreviations as in Table } 1 \text {. }\end{array}$} \\
\hline
\end{tabular}


Table 4

Re-read variability of $T_{1}$ mapping measurements.

\begin{tabular}{|c|c|c|c|}
\hline Sequence & Region & Intra-observer ICC (95\% Cl) & Inter-observer ICC (95\% Cl) \\
\hline \multirow[t]{2}{*}{ MOLLI } & Average mid & $0.989(0.91-0.99)$ & $0.968(0.76-0.99)$ \\
\hline & Septal ROI & $0.978(0.78-0.99)$ & $0.950(0.53-0.99)$ \\
\hline \multirow[t]{2}{*}{ Diastolic SAPPHIRE } & Average mid & $0.996(0.45-0.99)$ & $0.993(082-0.99)$ \\
\hline & Septal ROI & $0.987(0.89-0.99)$ & $0.954(0.52-0.99)$ \\
\hline \multirow[t]{2}{*}{ Systolic SAPPHIRE } & Average mid & $0.957(0.64-0.99)$ & $0.953(0.54-0.99)$ \\
\hline & Septal ROI & $0.964(0.66-0.99)$ & $0.982(0.87-0.99)$ \\
\hline \multirow[t]{2}{*}{ Oth order DB SAPPHIRE } & Average mid & $0.994(0.95-0.99)$ & $0.984(0.50-0.99)$ \\
\hline & Septal ROI & $0.991(0.76-0.99)$ & $0.986(0.84-0.99)$ \\
\hline \multirow[t]{2}{*}{ 2nd order DB SAPPHIRE } & Average mid & $0.973(0.78-0.99)$ & $0.967(0.47-0.99)$ \\
\hline & Septal ROI & $0.994(0.79-0.99)$ & $0.992(0.88-0.99)$ \\
\hline \multicolumn{4}{|c|}{$\begin{array}{l}\text { Agreement was considered excellent when ICC }>0.74 \text {, good when ICC }=0.60-0.74 \text {, fair when ICC }= \\
0.40-0.59 \text { and poor when ICC }<0.4 \\
C l \text {, confidence interval; ICC, intraclass correlation coefficient. Other abbreviations as in Tables } 1 \text { and } 2\end{array}$} \\
\hline
\end{tabular}

\section{Figures}




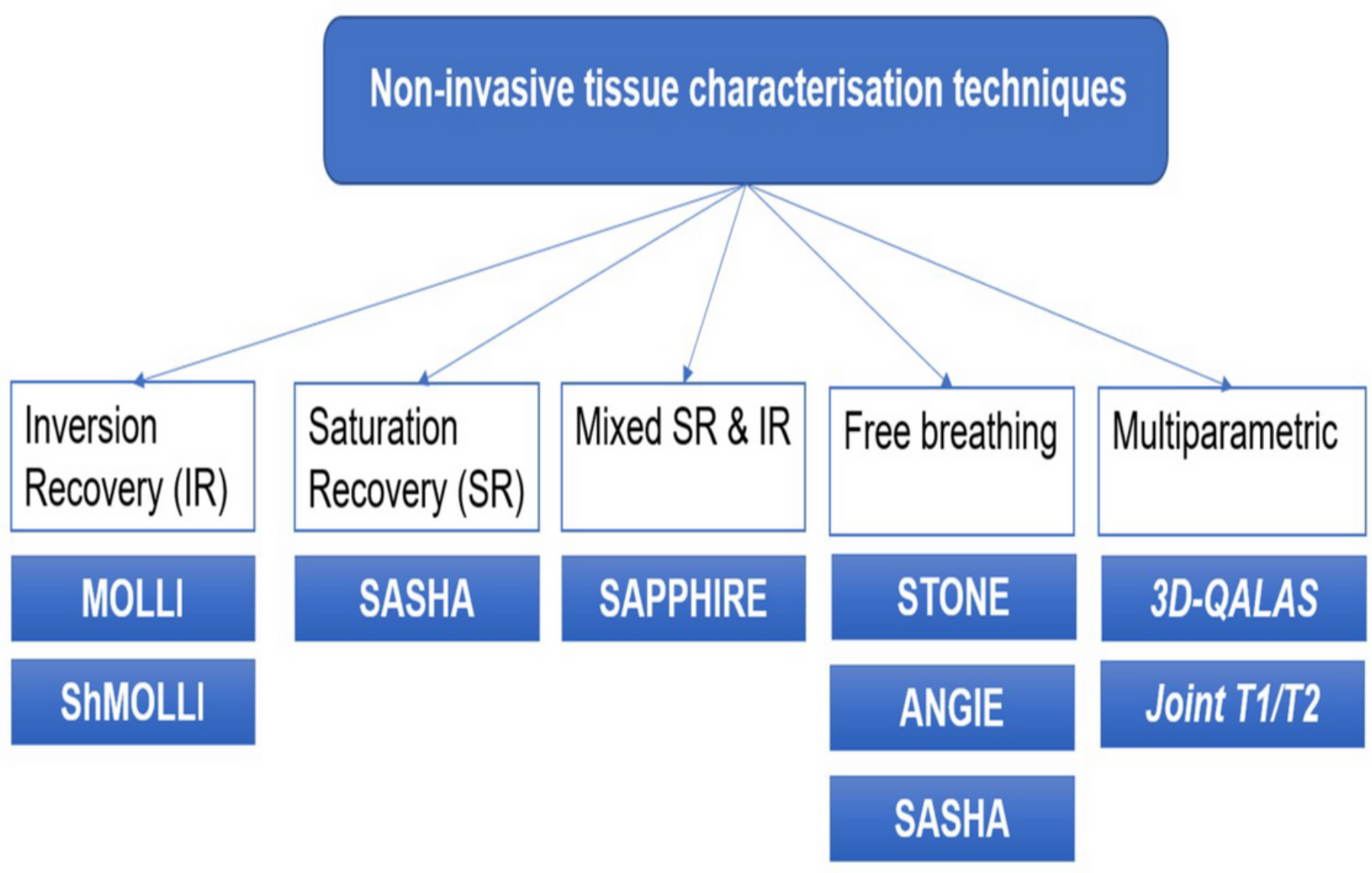

Figure 1

Basic overview of T1 mapping acquisition strategies. ANGIE, Accelerated and Navigator-Gated LookLocker Imaging for Cardiac T1 Estimation; 3D-QALAS, three-dimensional-QuAntification using an interleaved Look-Locker Acquisition Sequence with T2 preparation pulse; MOLLI, Modified Look-Locker Inversion recovery; Prep, preparation; SAPPHIRE, Saturation Pulse Prepared Heart-Rate Independent Inversion REcovery Sequence; SASHA, saturation recovery single shot acquisition; SAT, saturation; Seg, segmented; ShMOLLI, shortened MOLLI; STONE, slice-interleaved T1 mapping sequence. 


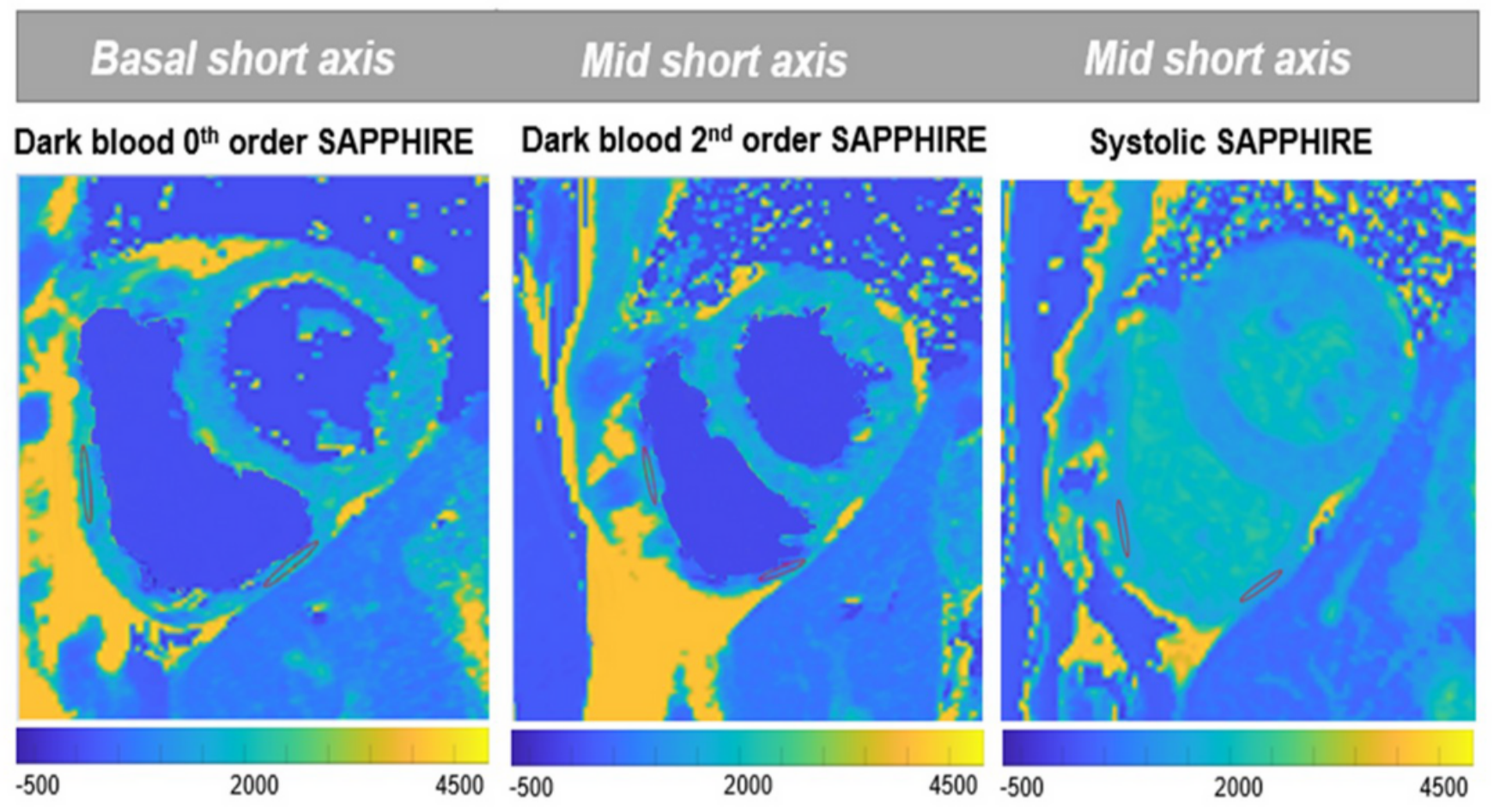

\section{Figure 2}

Right ventricular (RV) T1 times were determined by manually tracing regions of interest on the short axis slices as follows: (a) Basal short axis slice: RV inferior wall or RV free wall; (b) Mid short axis slice: RV inferior wall and RV free wall. Exemplar RV ROIs at 1.5T are shown for 3 study participants. Other abbreviations as in Figure 1. 


\section{a) Bright blood diastolic SAPPHIRE}

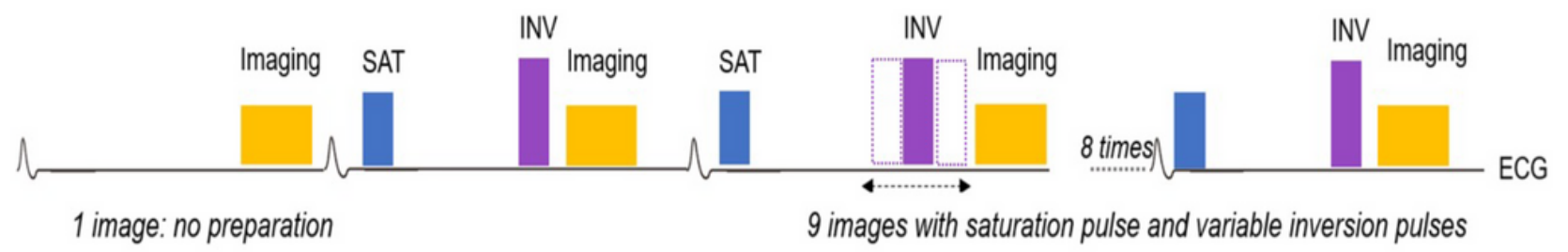

\section{b) Bright blood systolic SAPPHIRE}

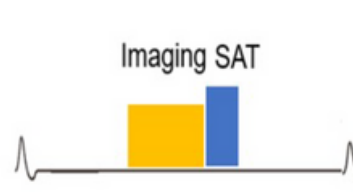

1 image: no preparation

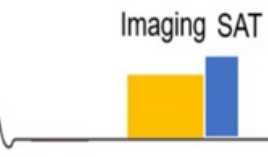

4 images with saturation pulse

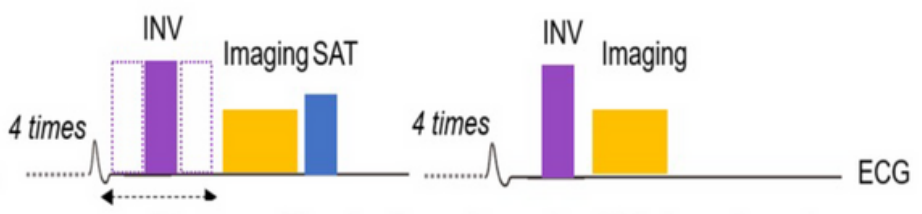

5 images with saturation pulse and variable inversion pulses

\section{Figure 3}

Sequence diagrams of the SAPPHIRE bright blood diastolic (a) and systolic (b) T1 mapping variants. ECG, Electrocardiogram; INV, Inversion pulse. Other abbreviations as in Figure 1.

a) Dark blood SAPPHIRE (0 $0^{\text {th }}$ order)
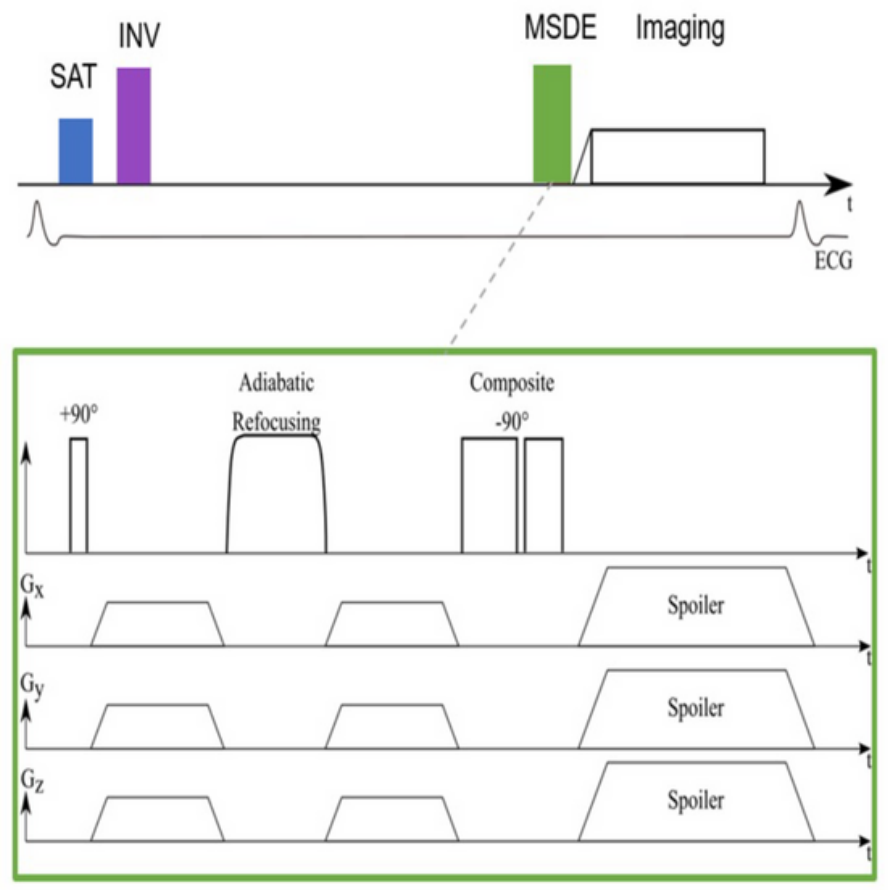

b) Dark blood SAPPHIRE (2nd order)
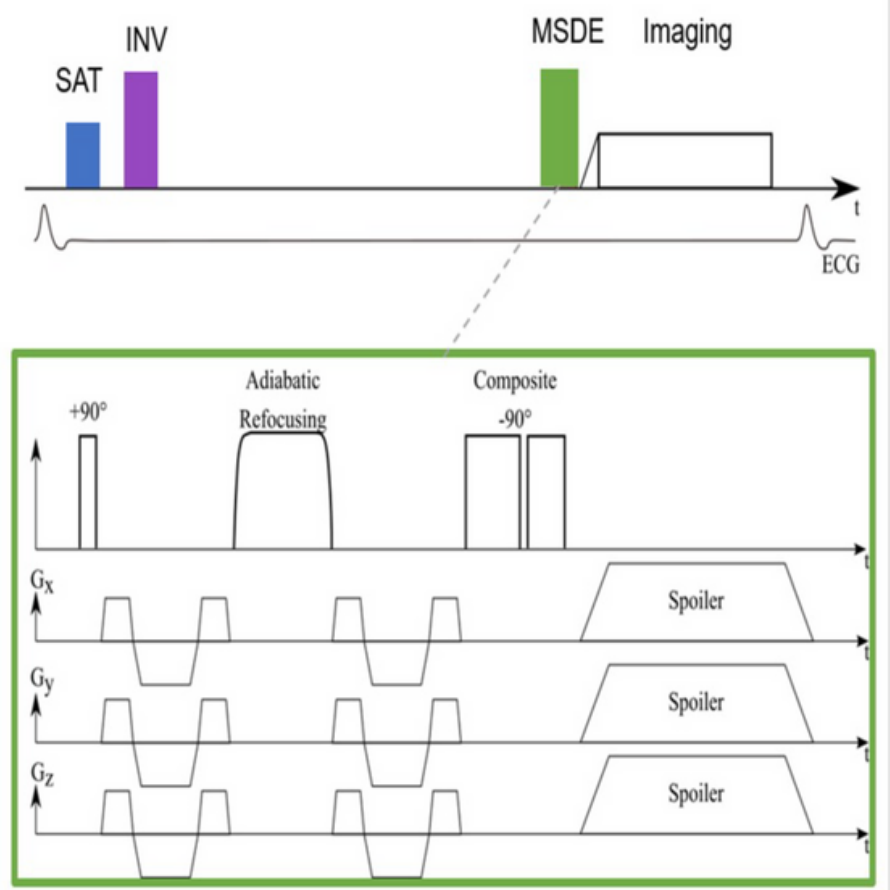


\section{Figure 4}

Sequence diagrams of the SAPPHIRE dark blood T 1 mapping variants with 0th (a) and 2nd (b) order flow sensizting gradients. MSDE, Motion-sensitized driven equilibrium. Other abbreviations as in Figures 1 and 3.
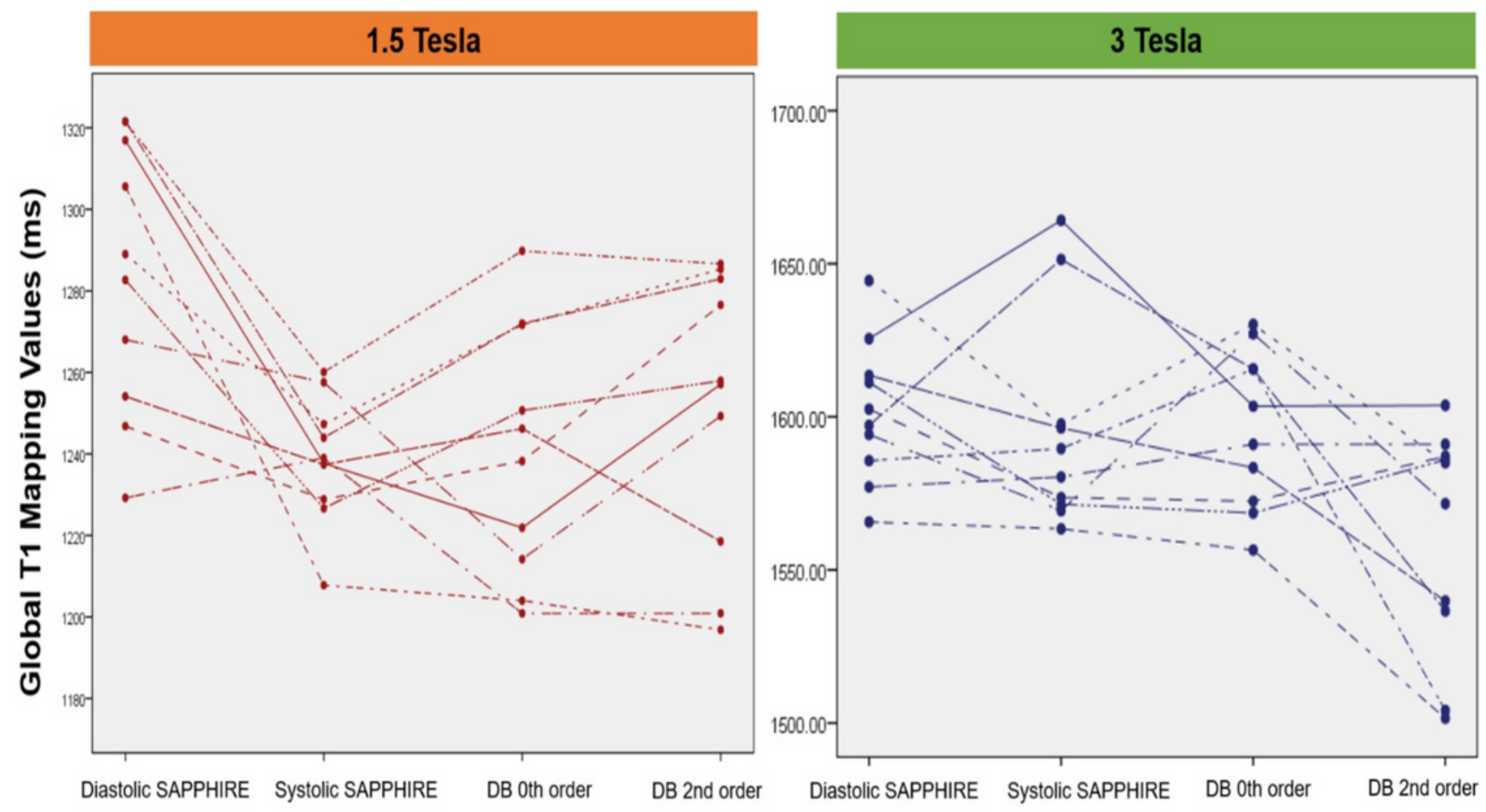

Figure 5

Profile plot displays the global T1 times obtained using the various SAPPHIRE variants in all ten healthy volunteers in our study. Each dot represents an individual. DB, Dark blood. Other abbreviations as in Figure 1. 

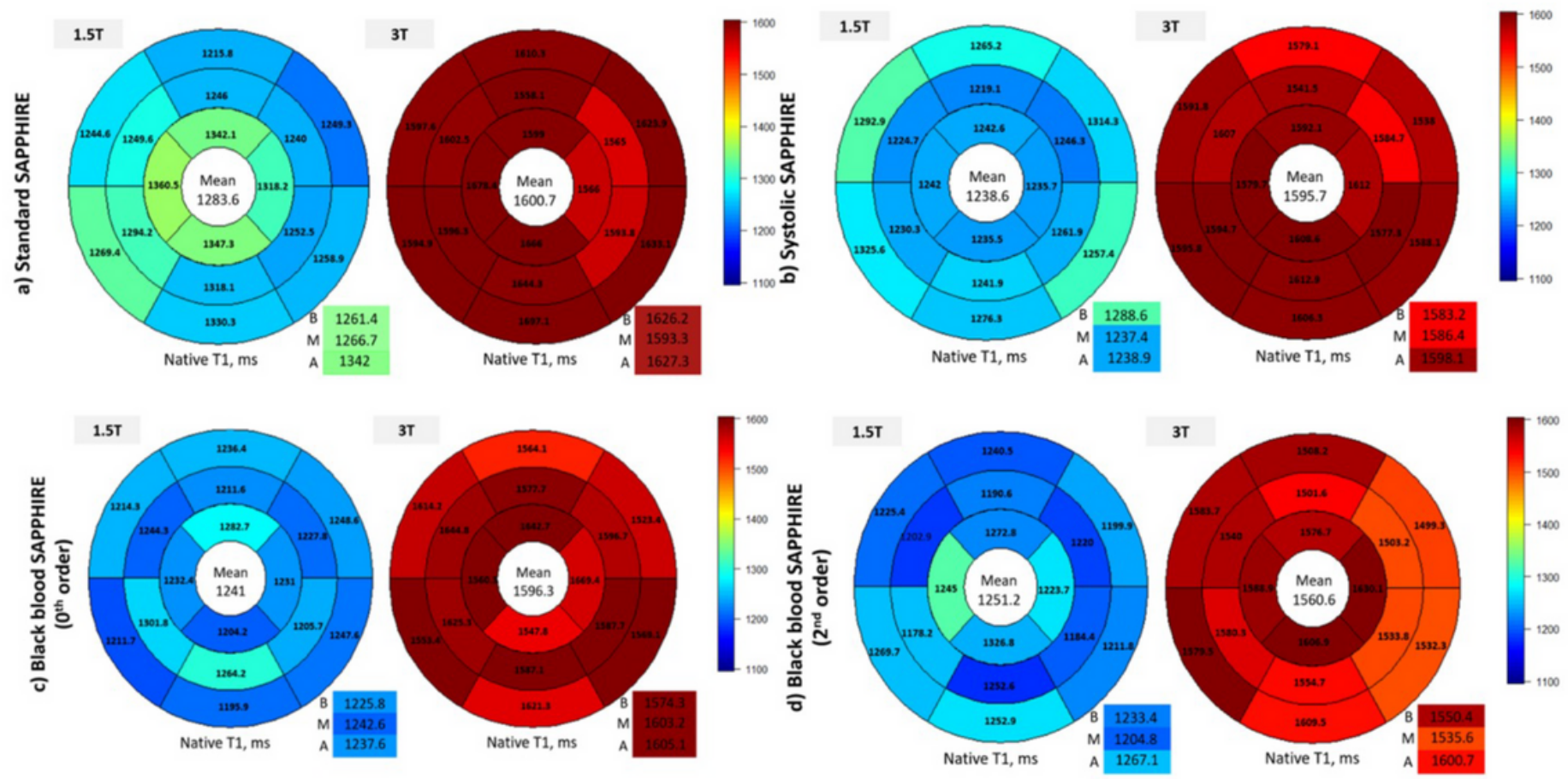

\section{Figure 6}

Bullseye plots illustrating the mean segmental T1 values of all healthy volunteers in three short-axis slices ( $B$, basal; $M$, mid-ventricular; $A$, apical) according to SAPPHIRE variant and field strength (1.5T blue, $3 \mathrm{~T}$ red). Global T1 is reported in the center of the bullseye and slice-specific means in the bottom right boxes. T, Tesla. Other abbreviations as in Figures 1 and 5. 
a) Global T1 values across sequences (including apical slice data).
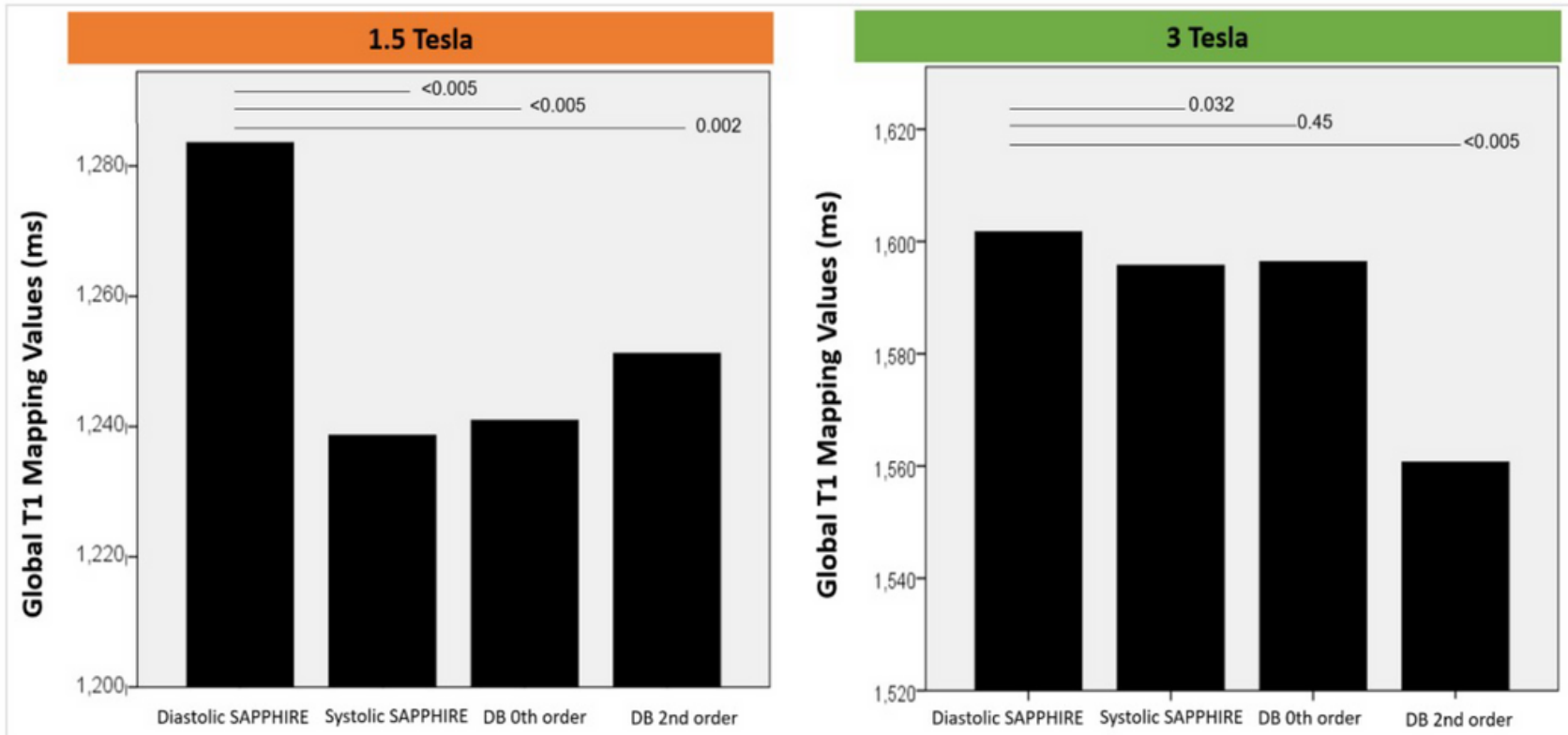

b) Global $T 1$ values across sequences (after excluding the apical slice)
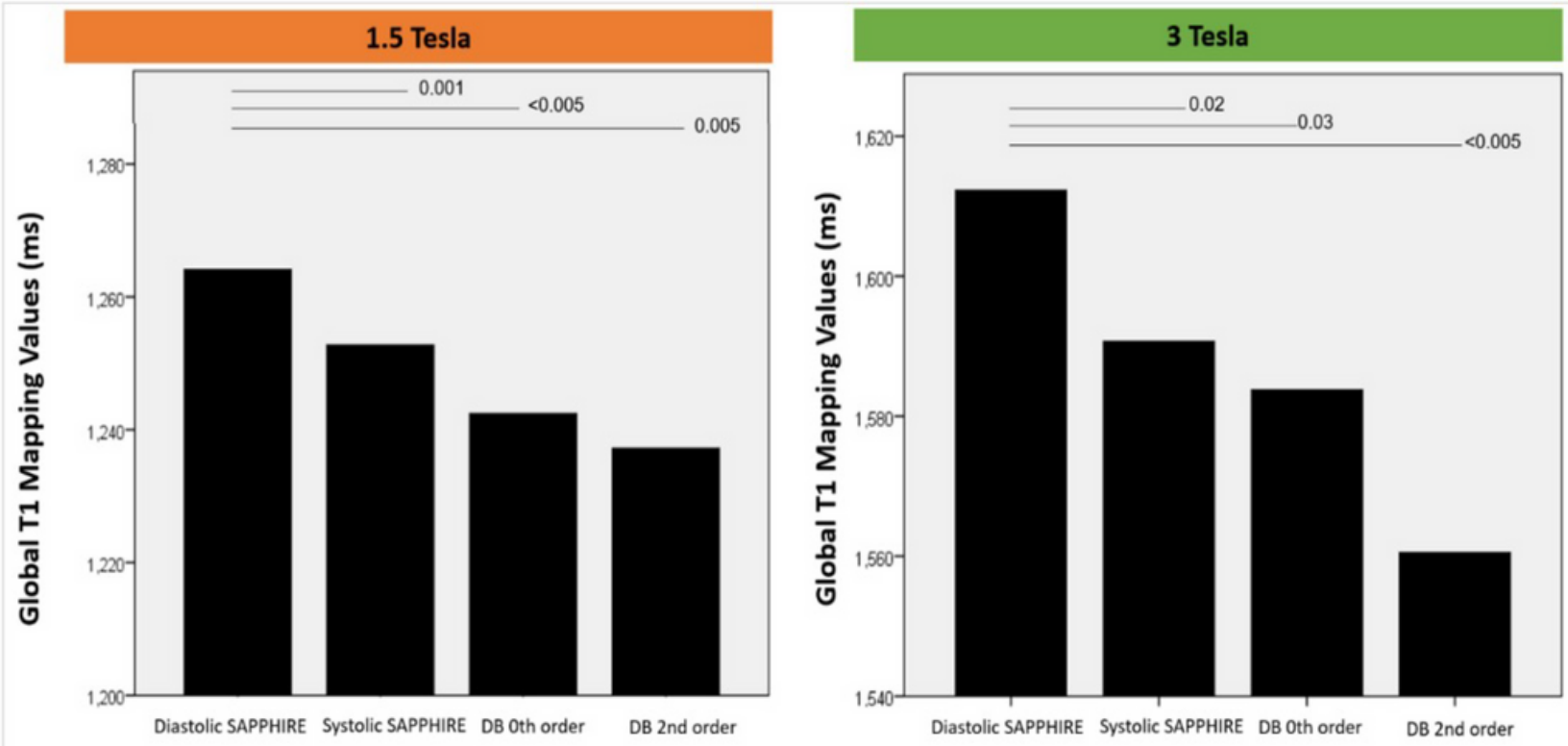

Figure 7

Global T1 mapping values at 1.5T (left) and 3T (right) by diastolic, systolic, 0th order and 2nd order SAPPHIRE sequences (a) including the apical slice (b) excluding the apical slice. Numbered lines above the bars represent $p$ values for differences obtained using mixed effect models. Abbreviations as in Figures 1,5 and 6 . 


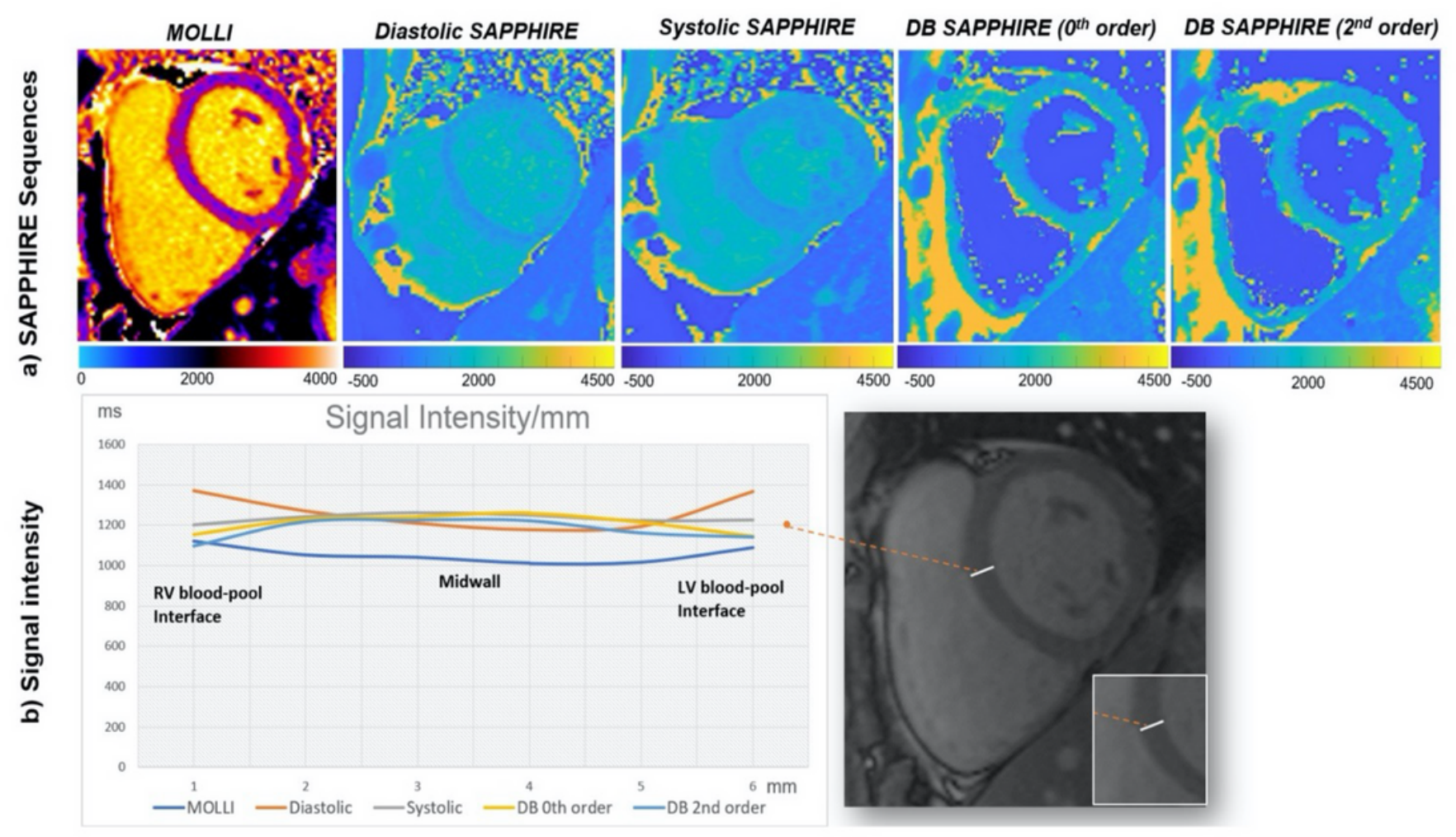

\section{Figure 8}

(a) Exemplar mid left ventricular short axis slice native T1 maps from the same participant by MOLLI and the 4 SAPPHIRE variants (diastolic, systolic, DB 0th and 2nd order) at 1.5T. (b) Averaged transmural 6point linear profiles (from the RV to LV side of the mid septum) of myocardial T1 times for all participants. LV, left ventricle. Other abbreviations as in Figures 1 and 5.

\section{Supplementary Files}

This is a list of supplementary files associated with this preprint. Click to download.

- Supplementarymaterial.docx 